\title{
A New Stability Criterion for Neutral Stochastic Delay Differential Equations with Markovian Switching
}

\author{
Wei Hu $(\mathbb{D})^{1,2}$ \\ ${ }^{1}$ School of Mathematics and Physics, Jiangsu University of Technology, Changzhou, 213001 Jiangsu, China \\ ${ }^{2}$ School of Mathematical Sciences and Institute of Finance and Statistics, Nanjing Normal University, Nanjing, 210023 Jiangsu, China \\ Correspondence should be addressed to Wei Hu; huw@jsut.edu.cn
}

Received 22 July 2018; Accepted 25 September 2018; Published 14 October 2018

Academic Editor: Ju H. Park

Copyright (c) 2018 Wei Hu. This is an open access article distributed under the Creative Commons Attribution License, which permits unrestricted use, distribution, and reproduction in any medium, provided the original work is properly cited.

In this short paper, a new stability theorem for neutral stochastic delay differential equations with Markovian switching is investigated by applying stochastic analysis technique and Razumikhin stability approach. A novel criterion of the pth moment exponential stability is derived for the related systems. The feature of the criterion shows that the estimated upper bound for the diffusion operator of Lyapunov function is allowed to be indefinite, even if to be unbounded, which can loosen the constraints of the existing results. Last, an example is provided to illustrate the usefulness and significance of the theoretical results.

\section{Introduction}

As is well-known, the stability is very hot topic in deterministic or stochastic dynamic systems (for instance, see [137] and references therein). Recently, a class of stochastic hybrid systems (also known as stochastic systems with Markovian switching) has been used to model many practical systems where they may experience abrupt changes in their structure and parameters. In [38], the author explained that the Markovian switching systems had been emerging as a convenient mathematical framework for the formulation of various design problems in different fields such as target tracking, fault tolerant control, and manufacturing processes, which can be seen as the motivation of wide practical use of the theoretic results for hybrid systems. Owing to their widely real applications, stochastic systems with Markovian switching have received a great deal of attention and many interesting results have been reported in the literature. For example, [39] considered the stability of stochastic differential equations with infinite Markovian switchings and [40] studied the stability for stochastic differential equations with semi-Markovian switchings. The results of stochastic hybrid systems are applied in feedback controls and neural networks; please refer to [41-45] and the reference therein.
As an important class of hybrid stochastic system, neutral stochastic delay differential equations with Markovian switching (NSDDEwMS) have been applied in practice, such as traffic control, neutral networks, and chemical process. Due to its wide applications, more and more researchers focus on this system. An important question for such a model is the stability analysis. Lyapunov-Razumikhin functions method and Lyapunov-Krasovskii functionals method are two effective methods that have been exploited for the stability analysis. For example, Mao in $[46,47]$ applied Razumikhin approach to derive the exponential stability criteria for neutral stochastic delay differential systems with Markovian switching. In [48-53], the authors considered the exponential stability by using Lyapunov-Krasovskii functionals method. Reference [54] applied some special techniques to study the exponential stability. Besides, Zhu in $[55,56]$ obtained several novel exponential stability criteria for some more complex systems with impulse control and Lévy noise. In addition, [57] considered the stability in distribution of neutral stochastic differential delay equations with Markovian switching and $[58,59]$ studied the almost sure stability for the same system.

Although the above exponential stability criteria can be used to judge the stability for many NSDDEwMS, nevertheless, these conditions in the above literature can be weakened 
to cover a large collection of NSDDEwMS. In other words, there are some shortcomings in the former criteria. The main is that all the above criteria for exponential stability in the related literature imposed some strict conditions on the diffusion operator of Lyapunov functions. For example, $[46,47]$ required the estimated upper bound for the diffusion operator of Lyapunov functions to be negative constant numbers. Reference [48] needed that the estimated upper bound $\lambda(t)$ must be a negative value function. Simply speaking, the former results all need that the upper bound $\lambda(t)$ for the diffusion operator of the Lyapunov function is negative definite for all $t$. This restriction leads to the criteria having strong conservativeness in practice due to the fact that there are a large number of time-varying systems not satisfying the above conditions. As shown by an example in Section 4 , the existing results and methods cannot be applied to analyse the stability for more general time-varying systems.

Motivated by the above discussion, in this short note, we focus on the exponential stability for NSDDEwMS. By using the notions such as uniformly stable function (USF), overshoot of the USF that dated from $[60,61]$ and then combining the stochastic analysis techniques, we obtain a novel stability criterion for NSDDEwMS. The feature of the criterion is that the coefficients of the estimated upper bound for the diffusion operator of Lyapunov functions can be allowed sign-changing as the time-varying, which can be used more widely than the existing results.

The rest of the paper is organized as follows. In Section 2, we introduce the model of NSDDEwMS and some preliminaries. The main result and its proof will be displayed in Section 3. An example is provided in Section 4. Finally, we conclude this paper with some general remarks in Section 5.

Throughout this short paper, let $\left(\Omega, \mathscr{F}_{,}\left\{\mathscr{F}_{t}\right\}_{t \geq 0}, \mathbb{P}\right)$ be a complete probability space with a natural filtration $\left\{\mathscr{F}_{t}\right\}_{t \geq 0}$ satisfying the usual condition (i.e., it is right continuous and $\mathscr{F}_{0}$ contains all $\mathbb{P}$-null sets). $|x|$ denotes the Euclidean norm of $x$. The symbol $C\left([-\tau, 0], \mathbb{R}^{n}\right)$ denotes the family of continuous function $\varphi$ from $[-\tau, 0]$ to $\mathbb{R}^{n}$ with the norm $\|\varphi\|=\sup _{-\tau \leq \theta \leq 0}|\varphi(\theta)|$. We use $C_{\mathscr{F}_{t}}^{p}\left([-\tau, 0], \mathbb{R}^{n}\right)$ to denote the family of all $\mathscr{F}_{t}$-measurable, $C\left([-\tau, 0], \mathbb{R}^{n}\right)$ valued random variables $\varphi$ satisfying $\sup _{-\tau \leq \theta \leq 0} \mathbb{E}|\varphi(\theta)|^{p}<\infty$. We use $\mathbb{E}[\cdot]$ to denote the expectation operator with respect to the probability measure $\mathbb{P}$. Let $B_{t}=B(t)=\left(B_{1}(t), B_{2}(t), \ldots, B_{m}(t)\right)^{T}$ be an $m$-dimensional Brownian motion defined on a complete probability space. $a \vee b=\max \{a, b\}$. $\mathbb{N}$ denotes the set of positive integers.

\section{Preliminaries}

Let $\{r(t), t \geq 0\}$ be a right-continuous Markov chain on the probability space $(\Omega, \mathscr{F}, \mathbb{P})$ taking values in a finite state space $\mathcal{S}=\{1,2, \ldots, N\}$ with generator $Q=\left(q_{i j}\right)_{N \times N}$ given by

$$
\begin{array}{r}
\mathbb{P}(r(t+\Delta t)=j \mid r(t)=i) \\
\quad= \begin{cases}q_{i j} \Delta t+o(\Delta t), & \text { if } i \neq j \\
1+q_{i i} \Delta t+o(\Delta t), & \text { if } i=j\end{cases}
\end{array}
$$

where $\Delta t>0$. Here $q_{i j} \geq 0$ is the transition rate from $i$ to $j$ if $i \neq j$ while $q_{i i}=-\sum_{j \neq i} q_{i j}=-q_{i}$.

In this short paper, we will consider the following neutral stochastic delay differential system with Markovian switching:

$$
\begin{aligned}
& d[x(t)-u(t, x(t-\tau), r(t))] \\
& =f(t, x(t), x(t-\tau), r(t)) d t \\
& \quad+g(t, x(t), x(t-\tau), r(t)) d B_{t}
\end{aligned}
$$

with the initial data $x_{0}=\phi=\{\phi(\theta),-\tau \leq \theta \leq$ $0\} \in C_{\mathscr{F}_{0}}^{p}\left([-\tau, 0], \mathbb{R}^{n}\right)$. We assume that $f, g$ satisfy the local Lipschitz condition and the linear growth condition. Additionally, we impose the following condition:

$$
|u(t, x, i)-u(t, y, i)| \leq \kappa|x-y|, \quad \kappa \in(0,1), i \in \mathcal{S}
$$

on $u(\cdot)$ to guarantee the existence and uniqueness of solution $x(t, \phi)$ for system (2) for all $t \geq 0$. We also assume that $f(t, 0,0, i)=0, g(t, 0,0, i)=0$, and $u(t, 0, i)=0$, which implies that the trivial solution of system (2) exists. In order to use Lyapunov's method to study the $p$ th moment exponential stability, we need the following definitions.

Definition 1. The trivial solution of (2) is said to be $p$ th moment exponentially stable if

$$
\limsup _{t \rightarrow \infty} \frac{\ln \mathbb{E}|x(t, \phi)|^{p}}{t}<0
$$

for all $\phi \in C_{\mathscr{F}_{0}}^{p}\left([-\tau, 0], \mathbb{R}^{n}\right)$. When $p=2,2$ th moment exponential stability is usually called mean square exponentially stable.

Definition 2. The function $V(t, x, i): \mathbb{R}^{+} \times \mathbb{R}^{n} \times \mathcal{S} \longrightarrow \mathbb{R}^{+}$ belongs to class $\Psi$ if it is a continuously twice differentiable with respect to $x$ and once differentiable with respect to $t$.

Next, we need an operator $\mathscr{L} V$ from $\mathbb{R}^{+} \times \mathbb{R}^{n} \times \mathbb{R}^{n} \times \mathcal{S}$ to $\mathbb{R}$ by

$$
\begin{aligned}
& \mathscr{L} V(t, x, y, i)=V_{t}(t, x-u(t, y, i), i)+V_{x}(t, x \\
& -u(t, y, i), i) f(t, x, y, i)+\frac{1}{2} \operatorname{trace}\left[g^{T}(t, x, y, i)\right. \\
& \left.\cdot V_{x x}(t, x-u(t, y, i), i) g(t, x, y, i)\right] \\
& +\sum_{j=1}^{N} q_{i j} V(t, x-u(t, y, i), j),
\end{aligned}
$$


where

$$
\begin{aligned}
& V_{x}(t, x, i) \\
& \quad=\left(\frac{\partial V(t, x, i)}{\partial x_{1}}, \frac{\partial V(t, x, i)}{\partial x_{2}}, \ldots, \frac{\partial V(t, x, i)}{\partial x_{n}}\right), \\
& V_{x x}(t, x, i)=\left(\frac{\partial^{2} V(t, x, i)}{\partial x_{i} \partial x_{j}}\right)_{n \times n}, \\
& V_{t}(t, x, i)=\frac{\partial V(t, x, i)}{\partial t} .
\end{aligned}
$$

In order to overcome the difficult caused by the neutral item, we need the following inequality.

Lemma 3. Let $p \geq 1$. Then

$$
\begin{aligned}
& (1-\kappa)^{p-1}|x|^{p}-\kappa(1-\kappa)^{p-1}|y|^{p} \leq|x-u(t, y, i)|^{p} \\
& \quad \leq(1+\kappa)^{p-1}\left(|x|^{p}+\kappa|y|^{p}\right)
\end{aligned}
$$

holds for any $(t, x, y, i) \in \mathbb{R}^{+} \times \mathbb{R}^{n} \times \mathbb{R}^{n} \times \mathcal{S}$.

The following lemma, which is important in the proof of the main result, can be found in Lemma 1 of [62].

Lemma 4. Let $T^{*}$ be a constant. Let a function $w$ : $\left[-T^{*}, \infty\right) \longrightarrow[0, \infty)$ admit a sequence $\left\{v_{i}\right\}$ and positive constants $\bar{v}_{a}$ and $\bar{v}_{b}$, such that $v_{0}=t_{0}, v_{i+1}-v_{i} \in\left[\bar{v}_{a}, \bar{v}_{b}\right]$ for all $i \geq 0 . w$ is continuous on $\left[v_{i}, v_{i+1}\right)$ for all $i \geq 0$ and the left limit $\lim _{t \rightarrow v_{i}^{-}} w(t)$ exist. Assume that there exists a constant $\rho \in(0,1)$ such that $w(t) \leq \rho \sup _{s \in\left[t-T^{*}, t\right]} w(s)$ holds for all $t \geq t_{0}$. Then

$$
w(t) \leq e^{\left((\ln \rho) / T^{*}\right)\left(t-t_{0}\right)} \sup _{s \in\left[t_{0}-T^{*}, t_{0}\right]} w(s)
$$

holds for all $t \geq t_{0}$.

The following definitions, which are dated from [60], are important in the proof of our main result.

Definition 5. A piecewise continuous function $\mu$ is said to be a USF if the following linear time-varying equation is globally uniformly asymptotically stable:

$$
d y(t)=\mu(t) y(t) d t, \quad \forall t \geq 0
$$

Definition 6. Let $\mu(t)$ be a USF. Then the set

$$
\Omega_{\mu}=\left\{T>0: \sup _{t \geq 0}\left\{\int_{t}^{t+T} \mu(s) d s\right\}<0\right\}
$$

is said to be the uniform convergence set of $\mu(t)$. For any $T \geq$ 0 , the overshoot $\varphi_{\mu}(T):=\varphi_{\mu}$ of $\mu(t)$ is defined as follows:

$$
\varphi_{\mu}=\sup _{t \geq 0}\left\{\max _{\theta \in[0, T]}\left\{\int_{t}^{t+\theta} \mu(s) d s\right\}\right\} .
$$

\section{Main Results}

In this section, we will use stochastic analysis theory, Lyapunov's method, and Lemmas 3 and 4 to obtain a criterion for $p$ th moment exponential stability of system (2). Our main result is the following.

Theorem 7. Let $p, c_{1}, c_{2}, \kappa, \tau$ be all positive numbers, and $\mu(t)$ is a USF. If there exist a function $V \in \Psi$ and a constant $T \in \Omega_{\mu}$ such that the following conditions hold for all $t \geq 0$,

(1) for all $x \in \mathbb{R}^{n}, i \in \mathcal{S}$,

$$
c_{1}|x|^{p} \leq V(t, x, i) \leq c_{2}|x|^{p},
$$

(2) the following Razumikhin-type condition holds:

$$
\begin{aligned}
& \mathbb{E} \mathscr{L} V(t, x(t), x(t-\tau), r(t)) \\
& \leq \mu(t) \mathbb{E} V(t, \tilde{x}(t), r(t)) \\
& \text { if } \min _{i \in S}\{\mathbb{E} V(t+\theta, x(t+\theta), i)\} \\
& \leq q \max _{i \in S}\{\mathbb{E} V(t, \tilde{x}(t), i)\} \\
& \quad \forall \theta \in[-\tau, 0], \text { where } q>\frac{c_{2}}{c_{1}} e^{\varphi_{\mu}}(1-\kappa)^{-p} .
\end{aligned}
$$

Then, system (2) is pth moment exponentially stable; here $\widetilde{x}(t) \triangleq x(t)-u(t, x(t-\tau), r(t))$.

Proof. First of all, we will prove the following fact: if $q^{-1} \mathbb{E} V(t+\theta, x(t+\theta), r(t)) \leq \mathbb{E} V(t, \widetilde{x}(t), r(t))$ holds for all $\theta \in[-\tau, 0]$ which implies $\mathbb{E} \mathscr{L} V(t, x(t), x(t-\tau), r(t)) \leq$ $\mu(t) \mathbb{E} V(t, \tilde{x}(t), r(t))$, then, for any $t>T$,

$$
\begin{aligned}
& \mathbb{E} V(t, \tilde{x}(t), r(t)) \\
& \leq \max \left\{\mathbb{E} V(t-T, \tilde{x}(t-T), r(t-T)) e^{\int_{t-T}^{t} \mu(s) d s},\right. \\
& q^{-1} \sup _{s \in[t-T, t]} \sup _{\theta \in[-\tau, 0]} \mathbb{E} V(s+\theta, x(s+\theta), r(s+\theta)) \\
& \left.\cdot e^{\varphi_{\mu}}\right\} .
\end{aligned}
$$

In order to prove inequality (14), we consider the following two cases:

(A): $\sup _{\theta \in[-\tau, 0]} \mathbb{E} V(s+\theta, x(s+\theta), r(s+\theta)) \leq q \mathbb{E} V(s, \widetilde{x}(s)$, $r(s))$ holds for all $s \in[t-T, t]$.

(B): $\sup _{\theta \in[-\tau, 0]} \mathbb{E} V(s+\theta, x(s+\theta), r(s+\theta)) \leq q \mathbb{E} V(s, \widetilde{x}(s)$, $r(s)$ ) does not hold for some $s \in[t-T, t]$.

For (A), we know that $\mathbb{E} \mathscr{L} V(s, x(s), x(s-\tau), r(s)) \leq$ $\mu(s) \mathbb{E} V(s, \widetilde{x}(s), r(s))$ holds for any $s \in[t-T, t]$. By using 
Ito's formula and the standard stopping times technique, we obtain that

$$
\begin{aligned}
& \mathbb{E}\left(e^{-\int_{t-T}^{t} \mu(s) d s} V(t, \tilde{x}(t), r(t))\right)=\mathbb{E} V(t-T, \tilde{x}(t \\
& \quad-T), r(t-T)) \\
& \quad+\mathbb{E} \int_{t-T}^{t}\left[-\mu(s) e^{-\int_{t-T}^{s} \mu(v) d v} V(s, \tilde{x}(s), r(s))\right. \\
& \left.\quad+e^{-\int_{t-T}^{s} \mu(v) d v} \mathscr{L} V(s, x(s), x(s-\tau), r(s))\right] d s \\
& \quad \leq \mathbb{E} V(t-T, \tilde{x}(t-T), r(t-T)),
\end{aligned}
$$

which implies that $\mathbb{E} V(t, \tilde{x}(t), r(t)) \leq \mathbb{E} V(t-T, \tilde{x}(t-T), r(t-$ T)) $e^{\int_{t-T}^{t} \mu(s) d s}$.

For (B), define $t^{*}=\sup \left\{s \in[t-T, t]: \sup _{\theta \in[-\tau, 0]} \mathbb{E} V(s+\right.$ $\theta, x(s+\theta), r(s+\theta))>q \mathbb{E} V(s, \widetilde{x}(s), r(s))\}$. Then $t^{*}<t$ or $t^{*}=t$. If $t^{*}<t$, then, for all $s \in\left[t^{*}, t\right], \sup _{\theta \in[-\tau, 0]} \mathbb{E} V(s+$ $\theta, x(s+\theta), r(s+\theta)) \leq q \mathbb{E} V(s, \widetilde{x}(s), r(s))$. So according to the result of $(\mathrm{A})$, we obtain that

$$
\begin{aligned}
& \mathbb{E} V(t, \tilde{x}(t), r(t)) \leq e^{\int_{t^{*}}^{t} \mu(s) d s} \mathbb{E} V\left(t^{*}, \tilde{x}\left(t^{*}\right), r\left(t^{*}\right)\right) \\
& \quad=e^{\int_{t^{*}}^{t} \mu(s) d s} q^{-1} \sup _{\theta \in[-\tau, 0]} \mathbb{E} V\left(t^{*}\right. \\
& \left.+\theta, x\left(t^{*}+\theta\right), r\left(t^{*}+\theta\right)\right) \\
& \quad \leq e^{\int_{t^{*}}^{t} \mu(s) d s} q^{-1} \sup _{s \in[t-T, t]} \sup _{\theta \in[-\tau, 0]} \mathbb{E} V(s \\
& +\theta, x(s+\theta), r(s+\theta)) \\
& \quad \leq e^{\varphi_{\mu}} q^{-1} \sup _{s \in[t-T, t]} \sup _{\theta \in[-\tau, 0]} \mathbb{E} V(s \\
& +\theta, x(s+\theta), r(s+\theta)) .
\end{aligned}
$$

If $t^{*}=t$, from the definition of $t^{*}$, we have

$$
\begin{aligned}
& \mathbb{E} V(t, \tilde{x}(t), r(t))=\mathbb{E} V\left(t^{*}, \tilde{x}\left(t^{*}\right), r\left(t^{*}\right)\right) \\
& \leq q^{-1} \sup _{\theta \in[-\tau, 0]} \mathbb{E} V\left(t^{*}+\theta, x\left(t^{*}+\theta\right), r\left(t^{*}+\theta\right)\right) \\
& \leq e^{\varphi_{\mu}} q^{-1} \sup _{s \in[t-T, t]} \sup _{\theta \in[-\tau, 0]} \mathbb{E} V(s+\theta, x(s+\theta), r(s+\theta)) .
\end{aligned}
$$

Combining the above three inequalities, we can see that inequality (14) holds.

Assume that $q^{-1} \mathbb{E} V(t+\theta, x(t+\theta), r(t)) \leq \mathbb{E} V(t, \tilde{x}(t), r(t))$ holds for all $\theta \in[-\tau, 0]$, then $q^{-1} \min _{i \in S}\{\mathbb{E} V(t+\theta, x(t+$ $\theta), i)\} \leq q^{-1} \mathbb{E} V(t+\theta, x(t+\theta), r(t)) \leq \mathbb{E} V(t, \tilde{x}(t), r(t)) \leq$ $\max _{i \in S}\{\mathbb{E} V(t, \tilde{x}(t), i)\}$ holds for all $\theta \in[-\tau, 0]$. According to condition (2), we know that $\mathbb{E} \mathscr{L} V(t, x(t), x(t-\tau), r(t)) \leq$ $\mu(t) \mathbb{E} V(t, \tilde{x}(t), r(t))$. Thus, from inequality (14), condition (1), and Lemma 3, we conclude that, for any $t>T$,

$$
\begin{aligned}
& \mathbb{E} V(t, \tilde{x}(t), r(t)) \\
& \leq \max \left\{\mathbb{E} V(t-T, \tilde{x}(t-T), r(t-T)) e^{\int_{t-T}^{t} \mu(s) d s},\right. \\
& q^{-1} \sup _{s \in[t-T, t]} \sup _{\theta \in[-\tau, 0]} \mathbb{E} V(s+\theta, x(s+\theta), r(s+\theta)) \\
& \left.\cdot e^{\varphi_{\mu}}\right\} \leq \max \left\{c_{2} \mathbb{E}|\tilde{x}(t-T)|^{p} e^{\int_{t-T}^{t} \mu(s) d s},\right. \\
& q^{-1} \sup _{s \in[t-T, t]} \sup _{\theta \in[-\tau, 0]} \mathbb{E} V(s+\theta, x(s+\theta), r(s+\theta)) \\
& \left.\cdot e^{\varphi_{\mu}}\right\} \leq \max \left\{c_{2}(1+\kappa)^{p-1}\right. \\
& \cdot\left(\mathbb{E}|x(t-T)|^{p}+\kappa \mathbb{E}|x(t-T-\tau)|^{p}\right) e^{\int_{t-T}^{t} \mu(s) d s}, \\
& q^{-1} \sup _{s \in[t-T, t]} \sup _{\theta \in[-\tau, 0]} \mathbb{E} V(s+\theta, x(s+\theta), r(s+\theta)) \\
& \left.\cdot e^{\varphi_{\mu}}\right\} \leq \max \left\{\frac{c_{2}}{c_{1}}(1+\kappa)^{p} e^{\int_{t-T}^{t} \mu(s) d s}, q^{-1} e^{\varphi_{\mu}}\right\} \\
& \cdot \sup _{s \in[t-T, t]} \sup _{\theta \in[-\tau, 0]} \mathbb{E} V(s+\theta, \\
& x(s+\theta), r(s+\theta)) .
\end{aligned}
$$

By condition (1), we can see that

$$
\begin{gathered}
\mathbb{E}|\widetilde{x}(t)|^{p} \leq \frac{c_{2}}{c_{1}} \max \left\{\frac{c_{2}}{c_{1}}(1+\kappa)^{p} e^{\int_{t-T}^{t} \mu(s) d s}, q^{-1} e^{\varphi_{\mu}}\right\} \\
\cdot \sup _{s \in[t-T, t]} \sup _{\theta \in[-\tau, 0]} \mathbb{E}|x(s+\theta)|^{p} \\
:=\eta \sup _{s \in[t-T, t]} \sup _{\theta \in[-\tau, 0]} \mathbb{E}|x(s+\theta)|^{p} .
\end{gathered}
$$

Using Lemma 3 again, we obtain that

$$
\begin{aligned}
\mathbb{E} & |x(t)|^{p} \\
\leq & {\left[\mathbb{E}|\widetilde{x}(t)|^{p}+\kappa(1-\kappa)^{p-1} \mathbb{E}|x(t-\tau)|^{p}\right](1-\kappa)^{1-p} } \\
& =(1-\kappa)^{1-p} \mathbb{E}|\widetilde{x}(t)|^{p}+\kappa \mathbb{E}|x(t-\tau)|^{p} \\
& \leq\left[(1-\kappa)^{1-p} \eta+\kappa\right] \sup _{s \in[t-T, t]} \sup _{\theta \in[-\tau, 0]} \mathbb{E}|x(s+\theta)|^{p} .
\end{aligned}
$$

In order to use Lemma 4 , we need to justify that $(1-\kappa)^{1-p} \eta+$ $\kappa<1$. In fact, on one hand, due to $q>\left(c_{2} / c_{1}\right) e^{\varphi_{\mu}}(1-\kappa)^{-p}$, there exists a constant $\rho \in(0,1)$ such that $\left(c_{2} / c_{1}\right) q^{-1} e^{\varphi_{\mu}}(1-$ $\kappa)^{1-p} \leq \rho-\kappa$. On the other hand, due to $\mu(t)$ being a USF and $T \in \Omega_{\mu}$, there exists a constant $\rho_{1} \in(0,1)$ such that 
$e^{\int_{t-T}^{t} \mu(s) d s}<\left(c_{1} / c_{2}\right)^{2}((1-\kappa) /(1+\kappa))^{p}\left(\left(\rho_{1}-\kappa\right) /(1-\kappa)\right)<1$. Thus

$$
\begin{aligned}
& \mathbb{E}|x(t)|^{p} \leq\left[(1-\kappa)^{1-p} \eta+\kappa\right] \\
& \cdot \sup _{s \in[t-T, t]} \sup _{\theta \in[-\tau, 0]} \mathbb{E}|x(s+\theta)|^{p}=\left[(1-\kappa)^{1-p} \frac{c_{2}}{c_{1}}\right. \\
& \left.\cdot \max \left\{\frac{c_{2}}{c_{1}}(1+\kappa)^{p} e^{\int_{t-T}^{t} \mu(s) d s}, q^{-1} e^{\varphi_{\mu}}\right\}+\kappa\right] \\
& \cdot \sup _{s \in[t-T, t]} \sup _{\theta \in[-\tau, 0]} \mathbb{E}|x(s+\theta)|^{p} \\
& \leq\left[\max _{\left.\left\{\rho_{1}-\kappa, \rho-\kappa\right\}+\kappa\right]}\right. \\
& \cdot \sup _{s \in[t-T, t]} \sup _{\theta \in[-\tau, 0]} \mathbb{E}|x(s+\theta)|^{p} \\
& \leq \rho^{*} \sup _{s \in[t-T, t]} \sup _{\theta \in[-\tau, 0]} \mathbb{E}|x(s+\theta)|^{p} \\
& \leq \rho^{*} \sup _{s \in[t-T-\tau, t]} \mathbb{E}|x(s)|^{p}
\end{aligned}
$$

where $\rho^{*}=\rho \vee \rho_{1} \in(0,1)$. By Lemma 4 , we derive that

$$
\mathbb{E}|x(t)|^{p} \leq e^{\left(\left(\ln \rho^{*}\right) /(T+\tau)\right)(t-T)} \sup _{s \in[-\tau, T]} \mathbb{E}|x(s)|^{p},
$$

which implies that

$$
\limsup _{t \rightarrow \infty} \frac{\ln \mathbb{E}|x(t)|^{p}}{t}=\frac{\ln \rho^{*}}{T+\tau}<0 .
$$

In other words, we have proved that system (2) is pth moment exponentially stable.

Remark 8. Here, by using stochastic analysis theory, some notions as uniformly stable function (USF), overshoot of the USF that dated from [60, 61], and some inequality techniques, we obtain a novel exponential stability criterion with respect to the related systems. The method we use here is rather different from the traditional Razumikhin approach. Thus, we provide a new method to investigate the stability analysis for neutral stochastic delayed differential equations with Markovian switchings. In the future, we can develop this approach to study the stability for impulsive neutral stochastic delayed differential equations.

Remark 9. If system (2) has no Markovian switching and $\mu(t) \equiv \mu<0$, then $e^{\varphi_{\mu}}=$; the condition of Theorem $7 q>$ $\left(c_{2} / c_{1}\right) e^{\varphi_{\mu}}(1-\kappa)^{-p}$ becomes $q>\left(c_{2} / c_{1}\right)(1-\kappa)^{-p}$, which is the same as the conditions in $[46,47]$. If the model we considered has no Markovian switching, stochastic disturbance, and neutral item, then we can obtain the same result as Theorem 1 in [60]. Thus, our result is the generalization of Theorem 1 in [60] and the main results in $[46,47]$.

Remark 10. In Theorem 3.1 of [53], the condition (3.3) ensured that the coefficient of the estimated upper bound for the diffusion operator of Lyapunov function is a negative value, and the condition of Theorem 3.1 in [48] required that the coefficient function of the estimated upper bound for the diffusion operator of Lyapunov function is a negative definite function, which are very conservative in the practice if the system is time-varying system. But our conditions allow this function to take values on $\mathbb{R}$ and even allow it to be unbounded. Thus, our result can be used more widely and is less conservative than the existing results. See Example 1 for more details.

\section{An Example}

In this section, we will give an example to illustrate the validity and significance of our result.

Example 1. Now we will consider the following neutraltype stochastic delay differential system with Markovian switching:

$$
\begin{aligned}
& d[x(t)-0.2 x(t-0.5)] \\
& =f(t, x(t), x(\mathrm{t}-0.5), r(t)) d t \\
& \quad+g(t, x(t), x(t-0.5), r(t)) d B_{t},
\end{aligned}
$$

where

$$
\begin{aligned}
& f(t, x(t), x(t-0.5), i) \\
& = \begin{cases}\left(\frac{1}{16} t \cos t^{2}-\frac{3}{2}\right) x(t-0.5)+\frac{1}{16} t \cos t^{2} x(t), & i=1, \\
\left(\frac{1}{8} t \cos t^{2}-1\right) x(t-0.5), & i=2,\end{cases}
\end{aligned}
$$

and

$$
\begin{aligned}
g(t, x(t), x(t-0.5), i) & \\
\quad & = \begin{cases}(\tanh t) x(t-0.5), & i=1, \\
e^{-t x^{2}(t-0.5)} x(t-0.5), & i=2 .\end{cases}
\end{aligned}
$$

$r(t)$ is a right-continuous Markov chain on the state space $\mathcal{S}=$ $\{1,2\}$ with the generator

$$
Q=\left[\begin{array}{cc}
-1 & 1 \\
2 & -2
\end{array}\right]
$$

Theorem 3.1 in [48] is void in determining the exponential stability for such a system. In fact, taking $V(t, x, i)=|x|^{2}$ and $c_{1}=c_{2}=1$, then, by Ito's formula,

$$
\begin{aligned}
& \mathbb{E} \mathscr{L} V(t, x(t), x(t-0.5), 1) \\
& \leq 2 \mathbb{E}\{(x(t)-0.2 x(t-0.5)) \\
& \left.\cdot\left[\left(\frac{1}{16} t \cos t^{2}-\frac{3}{2}\right) x(t-0.5)+\frac{1}{16} t \cos t^{2} x(t)\right]\right\} \\
& +\mathbb{E}|x(t-0.5)|^{2} \leq\left(\frac{7}{40} t \cos t^{2}-\frac{3}{4}\right) \mathbb{E}|x(t)|^{2} \\
& +\left(\frac{3}{40} t \cos t^{2}-\frac{1}{20}\right) \mathbb{E}|x(t-0.5)|^{2}
\end{aligned}
$$


and

$$
\begin{aligned}
& \mathbb{E} \mathscr{L} V(t, x(t), x(t-0.5), 2) \\
& \leq 2 \mathbb{E}\left[(x(t)-0.2 x(t-0.5))\left(\frac{1}{8} t \cos t^{2}-1\right)\right. \\
& \cdot x(t-0.5)]+\mathbb{E}|x(t-0.5)|^{2} \leq\left(\frac{1}{8} t \cos t^{2}-1\right) \\
& \cdot \mathbb{E}|x(t)|^{2}+\left(\frac{1}{10} t \cos t^{2}-\frac{2}{5}\right) \mathbb{E}|x(t-0.5)|^{2} .
\end{aligned}
$$

Obviously, the conditions in Theorem 3.1 [48] do not hold. But we can judge the exponential stability for such system by using our result. Take $V(t, x, i)=|x|^{2}$ and $c_{1}=c_{2}=1$. By Ito's formula,

$$
\begin{aligned}
\mathbb{E} \mathscr{L} V & (t, x(t), x(t-0.5), 1) \\
\leq & \left(\frac{1}{8} t \cos t^{2}-3\right) \mathbb{E}|\tilde{x}(t) x(t-0.5)| \\
& +\frac{1}{8} t \cos t^{2} \mathbb{E}|x(t) \tilde{x}(t)|+\mathbb{E}|x(t-0.5)|^{2} \\
\leq & {\left[\left(\frac{1}{8} t \cos t^{2}-3\right) \frac{q+1}{2}+q\right] \mathbb{E}|\tilde{x}(t)|^{2} } \\
& +\frac{1}{8} t \cos t^{2} \mathbb{E}|x(t) \tilde{x}(t)| \\
\leq & {\left[\left(\frac{1}{8} t \cos t^{2}-3\right) \frac{q+1}{2}+q\right] \mathbb{E}|\tilde{x}(t)|^{2} } \\
& +\frac{1}{8}\left(\frac{q+1}{2}\right) t \cos t^{2} \mathbb{E}|\tilde{x}(t)|^{2} \\
= & {\left[\frac{1}{8} t \cos t^{2}(q+1)-\frac{3(q+1)}{2}+q\right] \mathbb{E}|\tilde{x}(t)|^{2}, }
\end{aligned}
$$

and

$$
\begin{aligned}
& \mathbb{E} \mathscr{L} V(t, x(t), x(t-0.5), 2) \\
& \leq\left(\frac{1}{8} t \cos t^{2}-1\right) \mathbb{E}|\tilde{x}(t)|^{2} \\
&+\left(\frac{1}{8} t \cos t^{2}-1\right) \mathbb{E}|x(t-0.5)|^{2} \\
&+\mathbb{E}|x(t-0.5)|^{2} \\
& \leq {\left[\left(\frac{1}{4} t \cos t^{2}-2\right) \frac{q+1}{2}+q\right] \mathbb{E}|\tilde{x}(t)|^{2} } \\
&=\left(\frac{1}{8} t \cos t^{2}(q+1)+q\right) \mathbb{E}|\tilde{x}(t)|^{2} .
\end{aligned}
$$

Choosing $q=3$, then $\mathbb{E} \mathscr{L} V(t, x(t), x(t-0.5), r(t)) \leq$ $\mu(t) \mathbb{E} V(t, \tilde{x}(t), r(t))$, where $\mu(t)=(1 / 2) t \cos t^{2}-1$. Here,

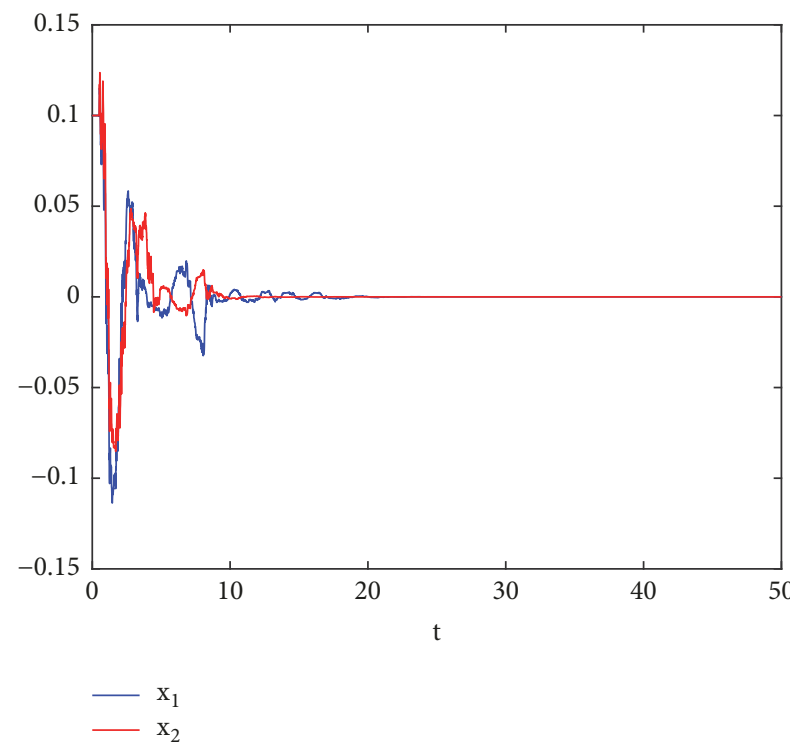

FIgURE 1: The state response of Example 1.

$\mu(t)$ is an unbounded function, taking values in $\mathbb{R}$, so we cannot use any former results to judge the mean-square exponential stability for such system. But, from our result, we can determine its stability. In fact, taking $T=1$, then $\int_{t}^{t+1} \mu(s) d s=-1 / 2<0$ holds for all $t \geq 0$. Thus $T \in$ $\Omega_{\mu}$. Additionally, $e^{\varphi_{\mu}} \leq e^{0.5}$, and $q^{-1} e^{\varphi_{\mu}}(1-k)^{-p} \leq 3^{-1} \times$ $e^{0.5} \times 0.8^{-2}<1$. By Theorem 7 , the system is mean square exponentially stable. See Figure 1.

\section{Conclusion}

In this paper, we investigate the stability criterion for neutral stochastic delay differential equations with Markovian switching. By using stochastic analysis technique and Razumikhin approach, we overcome the difficulty caused by the neutral item for the related system. Finally, we derive a new and novel Razumikhin exponential stability criterion. The feature of the result is that the estimated upper bound for the diffusion operator of Lyapunov function is allowed to take values on $\mathbb{R}$, even if it is allowed to be unbounded. The criterion can reduce some restrictiveness of the related results that are existing in the previous literature. An example is provided to show the superiority of the new exponential stability criterion.

\section{Data Availability}

No data were used to support this study.

\section{Conflicts of Interest}

The author declares that they have no conflicts of interest.

\section{Acknowledgments}

This work was supported by the China Postdoctoral Science Foundation (2018M632325). 


\section{References}

[1] X. Zheng, Y. Shang, and X. Peng, "Orbital stability of periodic traveling wave solutions to the generalized Zakharov equations," Acta Mathematica Scientia, vol. 37, no. 4, pp. 998-1018, 2017.

[2] Y. Guo, "Mean square exponential stability of stochastic delay cellular neural networks," Electronic Journal of Qualitative Theory of Differential Equations, no. 34, pp. 1-10, 2013.

[3] Q. Zhu and H. Wang, "Output feedback stabilization of stochastic feedforward systems with unknown control coefficients and unknown output function," Automatica, vol. 87, pp. 166-175, 2018.

[4] H. Wang and Q. Zhu, "Finite-time stabilization of high-order stochastic nonlinear systems in strict-feedback form," Automatica, vol. 54, pp. 284-291, 2015.

[5] G. Wang, "Existence-stability theorems for strong vector setvalued equilibrium problems in reflexive Banach spaces," Journal of Inequalities and Applications, vol. 239, pp. 1-14, 2015.

[6] Y. Bai and X. Mu, "Global asymptotic stability of a generalized SIRS epidemic model with transfer from infectious to susceptible," Journal of Applied Analysis and Computation, vol. 8, no. 2, pp. 402-412, 2018.

[7] Q. Zhu, S. Song, and T. Tang, "Mean square exponential stability of stochastic nonlinear delay systems," International Journal of Control, vol. 90, no. 11, pp. 2384-2393, 2017.

[8] Y. Guo, "Global asymptotic stability analysis for integrodifferential systems modeling neural networks with delays," Zeitschrift für Angewandte Mathematik und Physik, vol. 61, no. 6, pp. 971-978, 2010.

[9] L. Gao, D. Wang, and G. Wang, "Further results on exponential stability for impulsive switched nonlinear time-delay systems with delayed impulse effects," Applied Mathematics and Computation, vol. 268, pp. 186-200, 2015.

[10] Y. Guo, "Exponential stability analysis of travelling waves solutions for nonlinear delayed cellular neural networks," Dynamical Systems, vol. 32, no. 4, pp. 490-503, 2017.

[11] Y. Li, Y. Sun, and F. Meng, "New criteria for exponential stability of switched time-varying systems with delays and nonlinear disturbances," Nonlinear Analysis: Hybrid Systems, vol. 26, pp. 284-291, 2017.

[12] B. Wang and Q. Zhu, "Stability analysis of Markov switched stochastic differential equations with both stable and unstable subsystems," Systems \& Control Letters, vol. 105, pp. 55-61, 2017.

[13] L. G. Wang, K. P. Xu, and Q. W. Liu, "On the stability of a mixed functional equation deriving from additive, quadratic and cubic mappings," Acta Mathematica Sinica, vol. 30, no. 6, pp. 10331049, 2014.

[14] Q. Zhu, S. Song, and P. Shi, "Effect of noise on the solutions of non-linear delay systems," IET Control Theory \& Applications, vol. 12, no. 13, pp. 1822-1829, 2018.

[15] G. Liu, S. Xu, Y. Wei, Z. Qi, and Z. Zhang, "New insight into reachable set estimation for uncertain singular time-delay systems," Applied Mathematics and Computation, vol. 320, pp. 769-780, 2018

[16] W. Sun, Y. Wang, and R. Yang, "L2 disturbance attenuation for a class of time delay Hamiltonian systems," Journal of Systems Science \& Complexity, vol. 24, no. 4, pp. 672-682, 2011.

[17] Q. Zhu, "Asymptotic stability in the pth moment for stochastic differential equations with Lévy noise," Journal of Mathematical Analysis and Applications, vol. 416, no. 1, pp. 126-142, 2014.
[18] Y. Guo, "Global stability analysis for a class of Cohen-Grossberg neural network models," Bulletin of the Korean Mathematical Society, vol. 49, no. 6, pp. 1193-1198, 2012.

[19] J. $\mathrm{Hu}$ and $\mathrm{A} . \mathrm{Xu}$, "On stability of F-Gorenstein flat categories," Algebra Colloquium, vol. 23, no. 2, pp. 251-262, 2016.

[20] W. W. Sun, "Stabilization analysis of time-delay Hamiltonian systems in the presence of saturation," Applied Mathematics and Computation, vol. 217, no. 23, pp. 9625-9634, 2011.

[21] H. Wang and Q. Zhu, "Global Stabilization of Stochastic Nonlinear Systems via C 1 and C $\infty$ Controllers," Institute of Electrical and Electronics Engineers Transactions on Automatic Control, vol. 62, no. 11, pp. 5880-5887, 2017.

[22] Y. Guo, "Mean square global asymptotic stability of stochastic recurrent neural networks with distributed delays," Applied Mathematics and Computation, vol. 215, no. 2, pp. 791-795, 2009.

[23] Q. Zhu, "Stability analysis of stochastic delay differential equations with Lévy noise," Systems \& Control Letters, vol. 118, pp. 62-68, 2018.

[24] W. Sun and L. Peng, "Observer-based robust adaptive control for uncertain stochastic Hamiltonian systems with state and input delays," Lithuanian Association of Nonlinear Analysts. Nonlinear Analysis: Modelling and Control, vol. 19, no. 4, pp. 626-645, 2014.

[25] W. Qi, Y. Kao, X. Gao, and Y. Wei, "Controller design for time-delay system with stochastic disturbance and actuator saturation via a new criterion," Applied Mathematics and Computation, vol. 320, pp. 535-546, 2018.

[26] L. G. Wang and B. Liu, "Fuzzy stability of a functional equation deriving from additive, quadratic, cubic and quartic functions," Acta Mathematica Sinica, vol. 55, no. 5, pp. 841-854, 2012.

[27] Q. Zhu and B. Song, "Exponential stability of impulsive nonlinear stochastic differential equations with mixed delays," Nonlinear Analysis: Real World Applications, vol. 12, no. 5, pp. 2851-2860, 2011.

[28] F. Li and Y. Bao, "Uniform stability of the solution for a memorytype elasticity system with nonhomogeneous boundary control condition," Journal of Dynamical and Control Systems, vol. 23, no. 2, pp. 301-315, 2017.

[29] Y.-H. Feng and C.-M. Liu, "Stability of steady-state solutions to Navier-Stokes-Poisson systems," Journal of Mathematical Analysis and Applications, vol. 462, no. 2, pp. 1679-1694, 2018.

[30] X. Zheng, Y. Shang, and X. Peng, "Orbital stability of solitary waves of the coupled Klein-Gordon-Zakharov equations," Mathematical Methods in the Applied Sciences, vol. 40, no. 7, pp. 2623-2633, 2017.

[31] T. Li and Y. F. Yang, "Stability of large steady-state solutions to non-isentropic Euler-Maxwell systems," Journal of Nanjing Normal University, vol. 40, no. 4, pp. 26-35, 2017.

[32] Y. Guo, "Globally robust stability analysis for stochastic CohenGrossberg neural networks with impulse and time-varying delays," Ukrainian Mathematical Journal, vol. 69, no. 8, pp. 1049-1060, 2017.

[33] Q. Zhu, J. Cao, and R. Rakkiyappan, "Exponential input-to-state stability of stochastic Cohen-Grossberg neural networks with mixed delays," Nonlinear Dynamics, vol. 79, no. 2, pp. 1085-1098, 2015.

[34] L. G. Wang and B. Liu, "The Hyers-Ulam stability of a functional equation deriving from quadratic and cubic functions in quasi$\beta$-normed spaces," Acta Mathematica Sinica, vol. 26, no. 12, pp. 2335-2348, 2010. 
[35] M. Li and J. Wang, "Exploring delayed Mittag-Leffler type matrix functions to study finite time stability of fractional delay differential equations," Applied Mathematics and Computation, vol. 324, pp. 254-265, 2018.

[36] Y. Guo, "Nontrivial periodic solutions of nonlinear functional differential systems with feedback control," Turkish Journal of Mathematics, vol. 34, no. 1, pp. 35-44, 2010.

[37] L. Li, F. Meng, and P. Ju, "Some new integral inequalities and their applications in studying the stability of nonlinear integrodifferential equations with time delay," Journal of Mathematical Analysis and Applications, vol. 377, no. 2, pp. 853-862, 2011.

[38] M. Mariton, Jump Linear Systems in Automatica Control, Marcel Dekker, 1990.

[39] R. Song and Q. Zhu, "Stability of linear stochastic delay differential equations with infinite Markovian switchings," International Journal of Robust and Nonlinear Control, vol. 28, no. 3, pp. 825837, 2018.

[40] B. Wang and Q. Zhu, "Stability analysis of semi-Markov switched stochastic systems," Automatica, vol. 94, pp. 72-80, 2018.

[41] Q. X. Zhu and Q. Y. Zhang, "Pth moment exponential stabilisation of hybrid stochastic differential equations by feedback controls based on discrete-time state observations with a time delay," IET Control Theory \& Applications, vol. 11, no. 12, pp. 1992-2003, 2017.

[42] Q. Zhu, R. Rakkiyappan, and A. Chandrasekar, "Stochastic stability of Markovian jump BAM neural networks with leakage delays and impulse control," Neurocomputing, vol. 136, pp. 136151, 2014.

[43] Q. Zhu and J. Cao, "Stability of Markovian jump neural networks with impulse control and time varying delays," Nonlinear Analysis: Real World Applications, vol. 13, no. 5, pp. 2259-2270, 2012.

[44] Y. Liu, J. H. Park, B. Guo, F. Fang, and F. Zhou, "Eventtriggered dissipative synchronization for Markovian jump neural networks with general transition probabilities," International Journal of Robust and Nonlinear Control, vol. 28, no. 13, pp. 3893-3908, 2018.

[45] R. Zhang, D. Zeng, J. H. Park, Y. Liu, and S. Zhong, "A new approach to stochastic stability of markovian neural networks with generalized transition rates," IEEE Transactions on Neural Networks and Learning Systems, pp. 1-12, 2018.

[46] X. Mao, "Exponential stability in mean square of neutral stochastic differential-functional equations," Systems \& Control Letters, vol. 26, no. 4, pp. 245-251, 1995.

[47] X. Mao, "Razumikhin-type theorems on exponential stability of neutral stochastic functional-differential equations," SIAM Journal on Mathematical Analysis, vol. 28, no. 2, pp. 389-401, 1997.

[48] H. Chen, P. Shi, and C.-C. Lim, "Stability analysis for neutral stochastic delay systems with Markovian switching," Systems \& Control Letters, vol. 110, pp. 38-48, 2017.

[49] Q. Zhu and J. Cao, “pth moment exponential synchronization for stochastic delayed Cohen-Grossberg neural networks with Markovian switching," Nonlinear Dynamics, vol. 67, no. 1, pp. 829-845, 2012.

[50] Y. Chen, W. X. Zheng, and A. Xue, "A new result on stability analysis for stochastic neutral systems," Automatica, vol. 46, no. 12, pp. 2100-2104, 2010.

[51] L. Shaikhet, "Some new aspects of Lyapunov-type theorems for stochastic differential equations of neutral type," SIAM Journal on Control and Optimization, vol. 48, no. 7, pp. 4481-4499, 2010.
[52] Q. Zhu and J. Cao, "Stability analysis of markovian jump stochastic BAM neural networks with impulse control and mixed time delays," IEEE Transactions on Neural Networks and Learning Systems, vol. 23, no. 3, pp. 467-479, 2012.

[53] Y. Xu and Z. He, "Exponential stability of neutral stochastic delay differential equations with Markovian switching," Applied Mathematics Letters, vol. 52, pp. 64-73, 2016.

[54] K. Liu and X. Xia, "On the exponential stability in mean square of neutral stochastic functional differential equations," Systems \& Control Letters, vol. 37, no. 4, pp. 207-215, 1999.

[55] Q. Zhu, "Razumikhin-type theorem for stochastic functional differential equations with Lévy noise and Markov switching," International Journal of Control, vol. 90, no. 8, pp. 1703-1712, 2017.

[56] Q. Zhu, "pth moment exponential stability of impulsive stochastic functional differential equations with Markovian switching," Journal of The Franklin Institute, vol. 351, no. 7, pp. 3965-3986, 2014.

[57] J. Bao, Z. Hou, and C. Yuan, "Stability in distribution of neutral stochastic differential delay equations with Markovian switching," Statistics \& Probability Letters, vol. 79, no. 15, pp. 1663-1673, 2009.

[58] X. Li and X. Mao, "A note on almost sure asymptotic stability of neutral stochastic delay differential equations with Markovian switching," Automatica, vol. 48, no. 9, pp. 2329-2334, 2012.

[59] X. Mao, Y. Shen, and C. Yuan, "Almost surely asymptotic stability of neutral stochastic differential delay equations with Markovian switching," Stochastic Processes and Their Applications, vol. 118, no. 8, pp. 1385-1406, 2008.

[60] B. Zhou and A. V. Egorov, "Razumikhin and Krasovskii stability theorems for time-varying time-delay systems," Automatica, vol. 71, pp. 281-291, 2016.

[61] B. Zhou and W. Luo, "Improved Razumikhin and Krasovskii stability criteria for time-varying stochastic time-delay systems," Automatica, vol. 89, pp. 382-391, 2018.

[62] F. Mazenc, M. Malisoff, and S.-I. Niculescu, "Stability analysis for systems with time-varying delay: Trajectory based approach," in Proceedings of the 54th IEEE Conference on Decision and Control (CDC '15), pp. 1811-1816, Osaka, Japan, December 2015. 


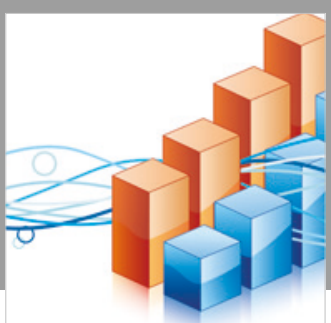

Advances in

Operations Research

\section{-n-m}
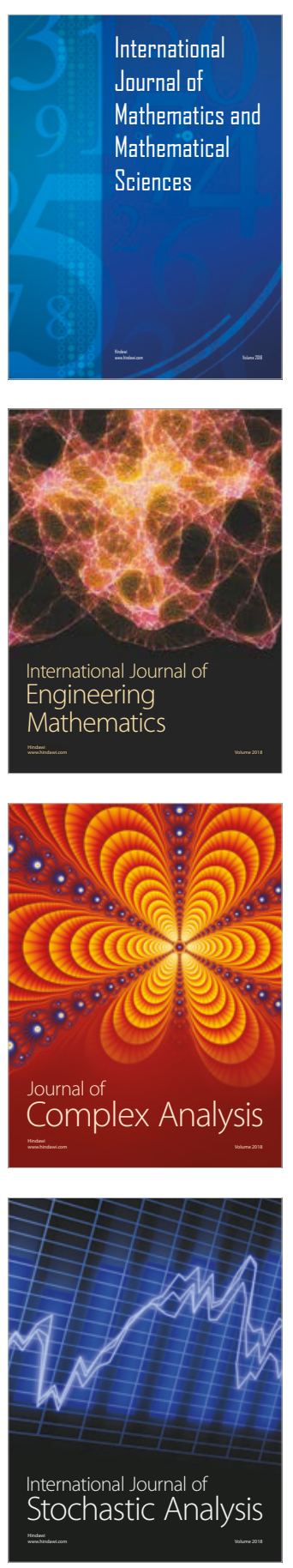
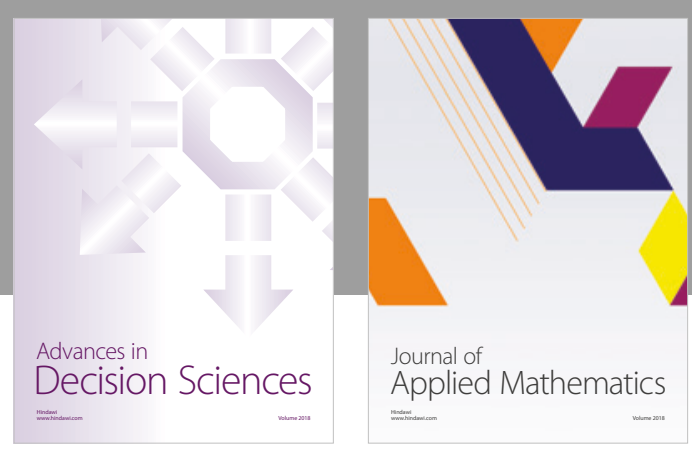

Journal of

Applied Mathematics
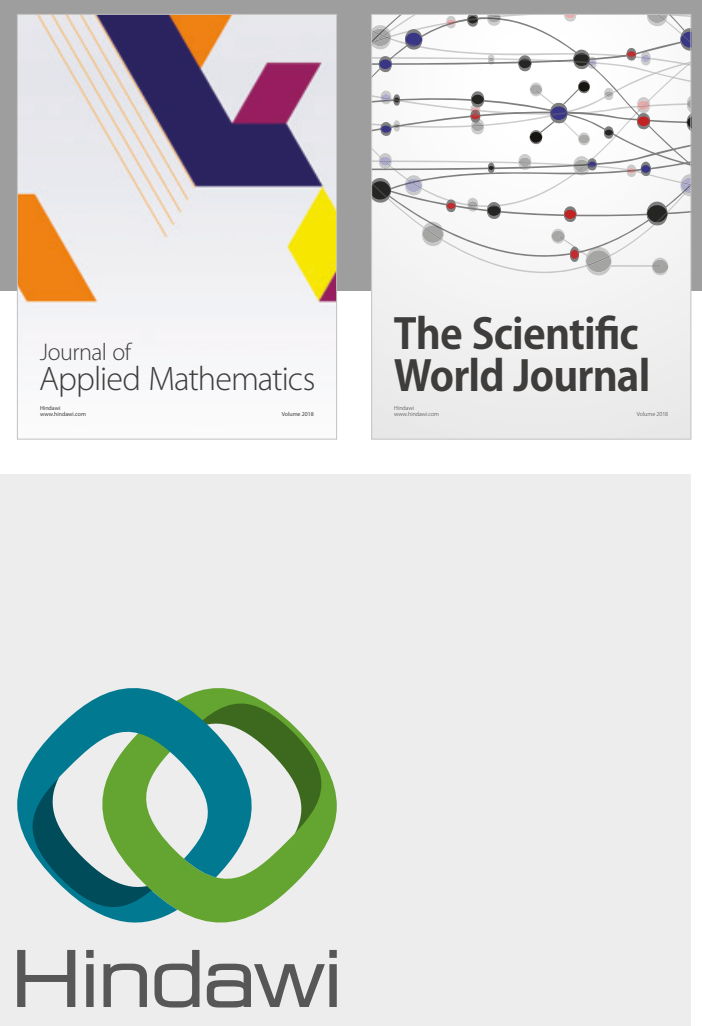

Submit your manuscripts at

www.hindawi.com

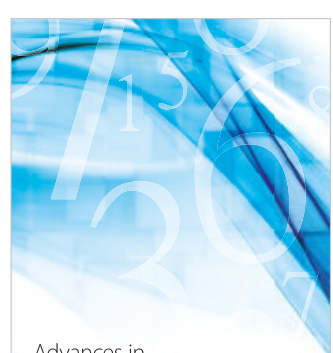

Advances in
Numerical Analysis
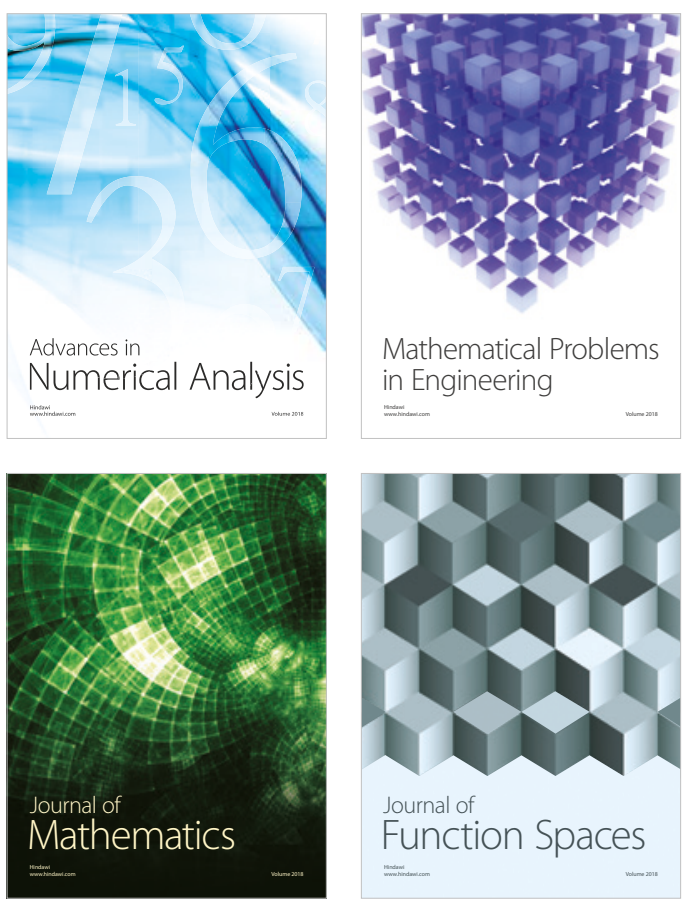

Mathematical Problems in Engineering

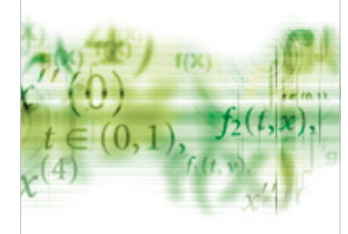

International Journal of

Differential Equations

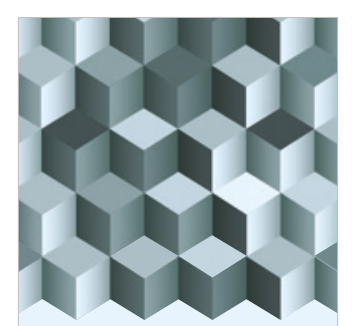

Journal of

Function Spaces

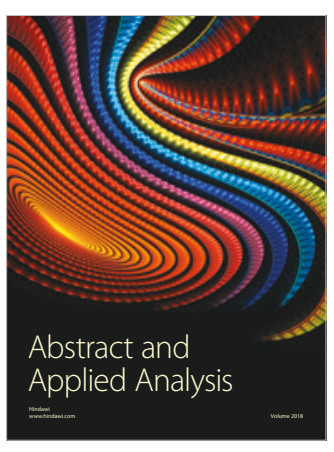

The Scientific

World Journal

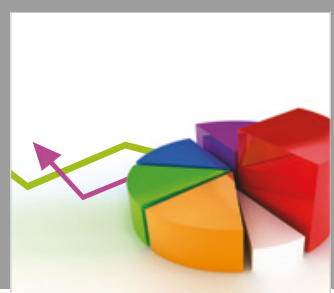

Journal of

Probability and Statistics
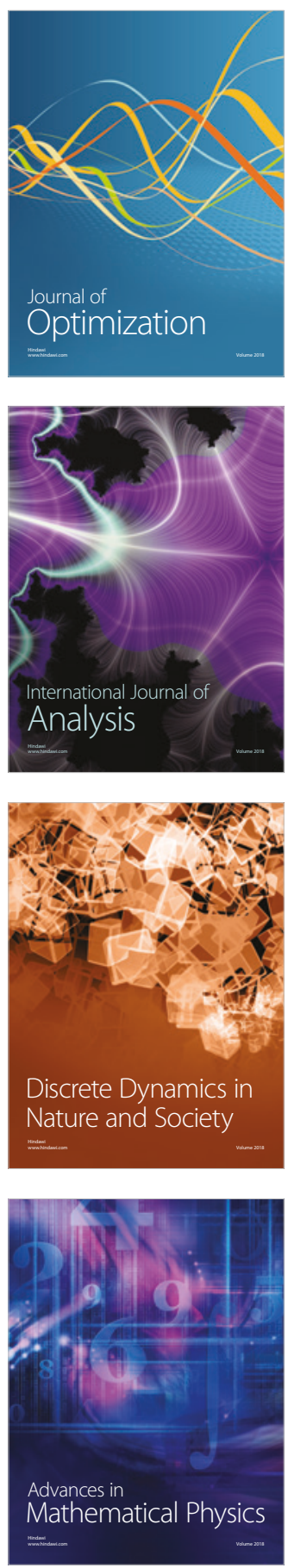